\title{
INEQUALITIES OF CORRELATION TYPE FOR SYMMETRIC STABLE RANDOM VECTORS
}

\author{
A. L. Koldobsky and S. J. Montgomery-Smith
}

\begin{abstract}
We point out a certain class of functions $f$ and $g$ for which random variables $f\left(X_{1}, \ldots, X_{m}\right)$ and $g\left(X_{m+1}, \ldots, X_{k}\right)$ are non-negatively correlated for any symmetric jointly stable random variables $X_{i}$. We also show another result that is related to the correlation problem for Gaussian measures of symmetric convex sets.
\end{abstract}

\section{INTRODUCTION}

For $0<q \leq 2$, let $Y$ be a symmetric $q$-stable random vector in $\mathbb{R}^{n}$ with characteristic function

$$
\phi(\theta)=\exp \left(-\left\|\sum_{i=1}^{n} \theta_{i} s_{i}\right\|^{q}\right), \quad \theta \in \mathbb{R}^{n},
$$

where $s_{1}, \ldots, s_{n} \in L_{q}([0,1])$, and the norm is taken from the space $L_{q}([0,1])$.

For any $k \in \mathbb{N}$, and any choice of vectors $\xi_{1}, \ldots, \xi_{k} \in \mathbb{R}^{n}$, the inner products $X_{1}=$ $\left(Y, \xi_{1}\right), \ldots, X_{k}=\left(Y, \xi_{k}\right)$ are symmetric $q$-stable random variables. The random variables $X_{1}, \ldots, X_{k}$ are jointly $q$-stable with zero mean, and we say that they are $\mathbb{R}^{n}$-generated in case we need to emphasize the dimension of the vector $Y$.

In this article, we show that, for any $m<k$, and any even continuous positive definite functions $f$ and $g$ on $\mathbb{R}^{m}$ and $\mathbb{R}^{k-m}$ respectively, the random variables $f\left(X_{1}, \ldots X_{m}\right)$ and $g\left(X_{m+1}, \ldots X_{k}\right)$ are non-negatively correlated, i.e.

$$
\mathbb{E}\left(f\left(X_{1}, \ldots, X_{m}\right) g\left(X_{m+1}, \ldots, X_{k}\right)\right) \geq \mathbb{E} f\left(X_{1}, \ldots X_{m}\right) \mathbb{E} g\left(X_{m+1}, \ldots, X_{k}\right),
$$

where $\mathbb{E}$ stands for the expectation.

Inequality (2) reminds one of some results related to the concept of associated random variables. Recall that random variables $X_{1}, \ldots, X_{k}$ are said to be associated if, for any choice of non-decreasing (in each variable) functions $f$ and $g$ on $\mathbb{R}^{k}$, the random variables $f\left(X_{1}, \ldots, X_{k}\right)$ and $g\left(X_{1}, \ldots, X_{k}\right)$ are non-negatively correlated whenever the expectations exist. Pitt (1982) proved that jointly Gaussian

1991 Mathematics Subject Classification. Primary 60E15. Secondary 60E07, 52A20, 42A82.

Key words and phrases. Stable random vector, Gaussian random vector, correlation, Fourier transform, positive definite function, convex set. 
random variables are associated if and only if the correlation between each pair is non-negative. Lee, Rachev and Samorodnitsky (1990) generalized this result to the case of jointly $q$-stable random variables by giving a necessary and sufficient condition in terms of the spectral measure. Inequality (2) points out a special class of functions $f$ and $g$ for which the correlation between $f(X)$ and $g(X)$ is non-negative independently of relations between the jointly $q$-stable random variables $X_{i}$. For other results related to association of random variables, see Joag-dev, Perlman and Pitt (1983), and Suquet (1994).

Another celebrated result of Pitt (1977) shows that, for any jointly Gaussian $\mathbb{R}^{2}$-generated random variables $X_{1}, \ldots, X_{k}$, inequality (2) holds if $f$ and $g$ are the indicator functions of cubes in $\mathbb{R}^{m}$ and $\mathbb{R}^{k-m}$, namely, for each $t>0$,

$$
P\left(\max _{1 \leq i \leq k}\left|X_{i}\right|<t\right) \geq P\left(\max _{1 \leq i \leq m}\left|X_{i}\right|<t\right) P\left(\max _{m+1 \leq i \leq k}\left|X_{i}\right|<t\right) .
$$

In other words, the quantity in the left-hand side is minimal (subject to the given marginal distributions) if for each choice of $i, j$ with $1 \leq i \leq m$ and $m+1 \leq j \leq k$ the random variables $X_{i}$ and $X_{j}$ are independent, that is to say, $b_{i j}=\operatorname{Cov}\left(X_{i}, X_{j}\right)=0$. An equivalent formulation of the same fact is that, for any symmetric convex sets $F$ and $G$ in $\mathbb{R}^{2}, \mu(F \cap G) \geq \mu(F) \mu(G)$, where $\mu$ is a symmetric Gaussian measure in $\mathbb{R}^{2}$. The question of whether the same is true for symmetric convex sets in $\mathbb{R}^{n}$ ( and, correspondingly, for $\mathbb{R}^{n}$-generated Gaussians) remains open (see Schlumprecht, Schechtman and Zinn (1994) for a historical survey and partial results).

In Section 3, we consider the quantity in the left-hand side of (3) as a function of the $m(k-m)$ variables $b_{i, j}$, and prove that, for every dimension $n$, this function has a local minimum at the origin. Note that, to solve the problem completely, one has to prove that the function has global minimum at the origin.

\section{A CORRELATiOn INEQUALity FOR POSITIVE}

\section{DEFINITE FUNCTIONS OF STABLE VARIABLES}

In order to prove inequality (2) we need the following simple fact.

Lemma 1. Let $0<q \leq 2$, and $\xi, \eta$ be any vectors from the space $L_{q}([0,1])$. Then

$$
\exp \left(-\|\xi+\eta\|^{q}\right)+\exp \left(-\|\xi-\eta\|^{q}\right) \geq 2 \exp \left(-\|\xi\|^{q}-\|\eta\|^{q}\right) .
$$

Proof. A result of W. Orlicz (1933) (see also Clarkson (1936)) states that, for every $0<q \leq 2$ and $\xi, \eta \in L_{q}$,

$$
\|\xi+\eta\|^{q}+\|\xi-\eta\|^{q} \leq 2\left(\|\xi\|^{q}+\|\eta\|^{q}\right) .
$$

Now use the inequality relating the arithmetic and geometric means to obtain

$$
\begin{aligned}
\exp \left(-\|\xi+\eta\|^{q}\right) & +\exp \left(-\|\xi-\eta\|^{q}\right) \geq \\
& 2 \exp \left(-\|\xi+\eta\|^{q} / 2-\|\xi-\eta\|^{q} / 2\right) \geq 2 \exp \left(-\|\xi\|^{q}-\|\eta\|^{q}\right)
\end{aligned}
$$


Theorem 1. Let $0<q \leq 2$ and $X_{1}, \ldots, X_{k}$ be jointly $q$-stable random variables. Then for any $m<k$ and any even continuous positive definite functions $f, g$ on $\mathbb{R}^{m}$ and $\mathbb{R}^{k-m}$ respectively, the random variables $f\left(X_{1}, \ldots, X_{m}\right)$ and $g\left(X_{m+1}, \ldots, X_{k}\right)$ are non-negatively correlated.

Proof. By Bochner's theorem, $f$ and $g$ are the characteristic functions of finite measures $\mu$ and $\nu$ on $\mathbb{R}^{m}$ and $\mathbb{R}^{k-m}$ respectively. The measures $\mu$ and $\nu$ are symmetric because the functions $f$ and $g$ are even.

Let $Y$ be the $q$-stable random vector in $\mathbb{R}^{n}$ generating $X_{1}, \ldots, X_{k}$, and let $\xi_{1}, \ldots, \xi_{k} \in \mathbb{R}^{n}$ be the vectors for which $X_{1}=\left(Y, \xi_{1}\right), \ldots, X_{k}=\left(Y, \xi_{k}\right)$. Denote by $\gamma$ the distribution of the vector $Y$, so $\gamma$ is a probability $q$-stable measure in $\mathbb{R}^{n}$ with the characteristic function given by (1).

Using Fubini's Theorem, we see that

$$
\begin{gathered}
\mathbb{E}\left(f\left(X_{1}, \ldots, X_{m}\right) g\left(X_{m+1}, \ldots, X_{k}\right)\right) \\
=\int_{\mathbb{R}^{n}} f\left(\left(x, \xi_{1}\right), \ldots,\left(x, \xi_{m}\right)\right) g\left(\left(x, \xi_{m+1}\right), \ldots,\left(x, \xi_{k}\right)\right) d \gamma(x) \\
=\int_{\mathbb{R}^{n}}\left(\int_{\mathbb{R}^{m}} \exp \left(-i\left(u_{1}\left(x, \xi_{1}\right)+\cdots+u_{m}\left(x, \xi_{m}\right)\right)\right) d \mu\left(u_{1}, \ldots, u_{m}\right) \times\right. \\
\left.\int_{\mathbb{R}^{k-m}} \exp \left(-i\left(u_{m+1}\left(x, \xi_{m+1}\right)+\cdots+u_{k}\left(x, \xi_{k}\right)\right)\right) d \nu\left(u_{m+1}, \ldots, u_{k}\right)\right) d \gamma(x)
\end{gathered}
$$

$=\int_{\mathbb{R}^{m}} \int_{\mathbb{R}^{k-m}}\left(\int_{\mathbb{R}^{n}} \exp \left(-i\left(x, \sum_{j=1}^{k} u_{j} \xi_{j}\right)\right) d \gamma(x)\right) d \mu\left(u_{1}, \ldots, u_{m}\right) d \nu\left(u_{m+1}, \ldots, u_{k}\right)$.

Let $\alpha=\sum_{j=1}^{m} u_{j} \xi_{j}, \beta=\sum_{j=m+1}^{k} u_{j} \xi_{j} \in \mathbb{R}^{n}$. Considering the coordinates of the vectors $\alpha$ and $\beta$ as linear functions of the coordinates of $u_{1}, \ldots, u_{m}$ and $u_{m+1}, \ldots, u_{k}$, respectively, and using (1) we see that the quantity in (4) is equal to

$$
I_{1}=\int_{\mathbb{R}^{m}} \int_{\mathbb{R}^{k-m}} \exp \left(-\left\|\sum_{j=1}^{n} \alpha_{j} s_{j}+\sum_{j=1}^{n} \beta_{j} s_{j}\right\|^{q}\right) d \mu\left(u_{1}, \ldots, u_{m}\right) d \nu\left(u_{m+1}, \ldots, u_{k}\right),
$$

where the norm is taken from the space $L_{q}([0,1])$. Denote by $I_{2}$ the expression in (5) with minus instead of plus under the norm. Since the measure $\nu$ is symmetric, $I_{1}=I_{2}$. By Lemma 1 ,

$$
\begin{gathered}
\left(I_{1}+I_{2}\right) / 2 \geq \int_{\mathbb{R}^{m}} \int_{\mathbb{R}^{k-m}} \exp \left(-\left\|\sum_{j=1}^{n} \alpha_{j} s_{j}\right\|^{q}\right) \times \\
\exp \left(-\left\|\sum^{n} \beta_{j} s_{j}\right\|^{q}\right) d \mu\left(u_{1}, \ldots, u_{m}\right) d \nu\left(u_{m+1}, \ldots, u_{k}\right)
\end{gathered}
$$




$$
\begin{gathered}
=\int_{\mathbb{R}^{m}} \exp \left(-\left\|\sum_{j=1}^{n} \alpha_{j} s_{j}\right\|^{q}\right) d \mu\left(u_{1}, \ldots, u_{m}\right) \times \\
\int_{\mathbb{R}^{k-m}} \exp \left(-\left\|\sum_{j=1}^{n} \beta_{j} s_{j}\right\|^{q}\right) d \nu\left(u_{m+1}, \ldots, u_{k}\right) .
\end{gathered}
$$

Repeating all the calculations in the reverse order we show that the latter quantity is equal to $\mathbb{E} f\left(X_{1}, \ldots X_{m}\right) \mathbb{E} g\left(X_{m+1}, \ldots, X_{k}\right)$ which finishes the proof.

Examples. (i) Let $\left.f\left(x_{1}, \ldots, x_{m}\right)=\left(1-\left|x_{1}\right|\right)_{+} \cdots\left(1-\left|x_{m}\right|\right)\right)_{+}$, and $g\left(x_{m+1}, \ldots, x_{k}\right)=$ $\left(1-\left|x_{m+1}\right|\right)_{+} \cdots\left(1-\left|x_{k}\right|\right)_{+}$, where the function $(1-|t|)_{+}$is equal to $1-|t|$ if $t \in[-1,1]$, and is equal to zero otherwise. It is well known that the function $(1-|t|)_{+}$is positive definite, and hence $f$ and $g$ are positive definite. Thus, by Theorem 1 , for every $m<k$ and every jointly stable random variables $X_{1}, \ldots, X_{k}$,

$$
\begin{gathered}
\mathbb{E}\left(\left(1-\left|X_{1}\right|\right)_{+} \cdot \ldots\left(1-\left|X_{k}\right|\right)_{+}\right) \geq \\
\mathbb{E}\left(\left(1-\left|X_{1}\right|\right)_{+} \cdot \ldots\left(1-\left|X_{m}\right|\right)_{+}\right) \mathbb{E}\left(\left(1-\left|X_{m+1}\right|\right)_{+} \cdot \ldots\left(1-\left|X_{k}\right|\right)_{+}\right) .
\end{gathered}
$$

The latter inequality can be generalized by taking any functions $f$ and $g$ of the form $f\left(x_{1}, \ldots, x_{m}\right)=f_{1}\left(x_{1}\right) \ldots f_{m}\left(x_{m}\right), g\left(x_{m+1}, \ldots, x_{k}\right)=f_{m+1}\left(x_{m+1}\right) \ldots f_{k}\left(x_{k}\right)$, where $f_{1}, \ldots, f_{k}$ are even functions on $\mathbb{R}$ which are convex and decreasing on $[0, \infty)$. Such functions $f_{i}$ are positive definite by a well-known result of Polya.

(ii) Let $q_{1}, \ldots, q_{k} \in(0,2], f\left(x_{1}, \ldots, x_{m}\right)=\exp \left(-\left|x_{1}\right|^{q_{1}}-\cdots-\left|x_{m}\right|^{q_{m}}\right)$, and $g\left(x_{m+1}, \ldots, x_{k}\right)=\exp \left(-\left|x_{m+1}\right|^{q_{m+1}}-\cdots-\left|x_{k}\right|^{q_{k}}\right)$. Since for any $q \in(0,2]$ the function $\exp \left(-|t|^{q}\right)$ is positive definite, it follows that $f$ and $g$ are positive definite. Therefore, for every $m<k$

$$
\begin{gathered}
\mathbb{E}\left(\exp \left(-\left|X_{1}\right|^{q_{1}}-\cdots-\left|X_{k}\right|^{q_{k}}\right)\right) \geq \\
\mathbb{E}\left(\exp \left(-\left|X_{1}\right|^{q_{1}}-\cdots-\left|X_{m}\right|^{q_{m}}\right)\right) \mathbb{E}\left(\exp \left(-\left|X_{m+1}\right|^{q_{m+1}}-\cdots-\left|X_{k}\right|^{q_{k}}\right)\right)
\end{gathered}
$$

Remarks. (i) In the case of jointly Gaussian random variables the result of Theorem 1 can be extended to some classes of continuous functions $f$ and $g$ with power growth at infinity and such that their Fourier transforms (in the sense of distributions) are non-negative locally integrable functions with power growth at infinity. To do that, consider the convolutions of the functions $f$ and $g$ with Gaussian densities $e_{n}$ approaching the $\delta$-function as $n \rightarrow \infty$, and slightly modify the proof of Theorem 1.

(ii) Y. Hu has recently proved that, for any even convex functions $f$ and $g$ on $\mathbb{R}^{n}$ and jointly Gaussian random variables $X_{1}, \ldots, X_{n}$, the random variables $f\left(X_{1}, \ldots, X_{n}\right)$ and $g\left(X_{1}, \ldots, X_{n}\right)$ are non-negatively correlated (private communication from T. Schlumprecht; compare the result of Hu with our Example 1). 


\section{ON THE LOCAL MiNimUM IN THE CORRELATION FOR}

GAUSSIAN MEASURES OF SYMMETRIC CONVEX SETS

Let $\nu$ be the standard symmetric Gaussian measure on $\mathbb{R}^{n}$. Is it true that

$$
\nu(F \cap G) \geq \nu(F) \nu(G)
$$

for all symmetric convex sets $F$ and $G$ in $\mathbb{R}^{n}$ ? In 1977, L. Pitt proved that the answer is positive in the case $n=2$. However, the question of whether the answer is positive for every dimension $n$ is still open.

It can be seen that it suffices to consider the sets $F=\left\{x \in \mathbb{R}^{n}:\left|\left(x, \xi_{1}\right)\right| \leq\right.$ $\left.1, \ldots,\left|\left(x, \xi_{k}\right)\right| \leq 1\right\}$ and $G=\left\{x \in \mathbb{R}^{n}:\left|\left(x, \xi_{k+1}\right)\right| \leq 1, \ldots,\left|\left(x, \xi_{2 k}\right)\right| \leq 1\right\}$, where $k$ is an integer, and $\xi_{1}, \ldots, \xi_{k}, \xi_{k+1}, \ldots, \xi_{2 k} \in \mathbb{R}^{n}$. For these sets $F$ and $G$, inequality (6) can be written in the form

$$
P\left(\max _{1 \leq i \leq 2 k}\left|X_{i}\right|<1\right) \geq P\left(\max _{1 \leq i \leq k}\left|X_{i}\right|<1\right) P\left(\max _{k+1 \leq i \leq 2 k}\left|X_{i}\right|<1\right)
$$

where $X_{1}, \ldots, X_{2 k}$ are the jointly Gaussian random variables generated by the vectors $\xi_{1}, \ldots, \xi_{k}, \xi_{k+1}, \ldots, \xi_{2 k} \in \mathbb{R}^{n}$ and a standard Gaussian random vector $Y$ in $\mathbb{R}^{n}$, so that $X_{i}=\left(Y, \xi_{i}\right)$ for each $i$.

It is easy to see that, to prove inequality (6), it suffices to consider the case where the vectors $\xi_{i}, i=1, \ldots, 2 k$ are linearly independent. For example, if $n<2 k$ and the system of vectors $\xi_{i}$ has rank $n$, we can transfer everything to the space $\mathbb{R}^{2 k}$, and consider the vectors $\eta_{i}=\xi_{i}+\epsilon e_{i} \in \mathbb{R}^{2 k}, i=1, \ldots, 2 k$ where, for each $i$, either $e_{i}=0$ or $\left\|e_{i}\right\|=1$ and $e_{i}$ is orthogonal to each of the vectors $\xi_{j}, j=1, \ldots, 2 k$ and $e_{j}, j \neq i$, so that the vectors $\eta_{i}$ are linearly independent in $\mathbb{R}^{2 k}$. Then inequality (7) for the random variables generated by the vectors $\eta_{i}$ would imply inequality

(7) for the random variables generated by $\xi_{i}$ 's by taking the limit as $\epsilon \rightarrow 0$ and applying the Lebesgue dominated convergence theorem.

Assume that the vectors $\xi_{i} \in \mathbb{R}^{2 k}, i=1, \ldots, 2 k$ are linearly independent. Then the joint distribution $\mu$ of random variables $X_{1}, \ldots, X_{2 k}$ is a non-singular Gaussian measure in $\mathbb{R}^{2 k}$, and the left-hand side of (7) is equal to

$$
P\left(\max _{1 \leq i \leq 2 k}\left|X_{i}\right|<1\right)=\mu\left([-1,1]^{2 k}\right) .
$$

We fix the scalar products $\left(\xi_{i}, \xi_{j}\right)$ for all choices of $i, j$ with either $1 \leq i, j \leq k$ or $k+1 \leq i, j \leq 2 k$, and consider the quantity $\mu\left([-1,1]^{2 k}\right)$ as a function of $k^{2}$ variables $b_{i, j}=\operatorname{Cov}\left(X_{i}, X_{j}\right), i=1, \ldots, k, j=k+1, \ldots, 2 k$. To prove Pitt's inequality, one has to show that this function has a global minimum at zero. Being unable to do that we show instead that the function has a local minimum at zero. This fact is a simple consequence of Theorem 2 below.

In the proof of Theorem 2 we use one result about log-concave functions. A non-negative function $f$ on $\mathbb{R}^{k}$ is called log-concave if, for every choice of $x, y \in \mathbb{R}^{k}$, and $0 \leq t \leq 1$ 
This means that the function $\log (f)$ is concave. Prekopa (1973) and Leindler (1972) have proved that if $f$ is a log-concave function on $\mathbb{R}^{k}$ and $0<m<k$, then the function

$$
g\left(x_{1}, \ldots, x_{m}\right)=\int_{\mathbb{R}^{k-m}} f\left(x_{1}, \ldots, x_{m}, z_{1}, \ldots, z_{k-m}\right) d z
$$

is also log-concave.

Theorem 2. Let $F$ and $G$ be symmetric convex sets in $\mathbb{R}^{k}$, and $\mu_{B}$ be a nonsingular probability Gaussian measure in $\mathbb{R}^{2 k}$ with the covariance matrix $\mathcal{A}=$ $\left[\begin{array}{cc}A & B \\ B^{T} & C\end{array}\right]$. Fix the $k \times k$ matrices $A$ and $C$, and consider $B=\left(b_{i, j}\right)_{i, j=1}^{k}$ as a variable from the space $\mathbb{R}^{k^{2}}$. Then the function $B \mapsto \mu_{B}(F \times G)$ has a local minimum at the point $B=0$.

Proof. Without loss of generality, we may suppose that $F$ and $G$ have compact closure. Let $\chi_{F}, \chi_{G}$ be the indicator functions of the sets $F$ and $G$. Taking Fourier transforms, we obtain

$$
\begin{gathered}
\mu_{B}(F \times G)=\int_{\mathbb{R}^{k}} \int_{\mathbb{R}^{k}} \chi_{F}(x) \chi_{G}(y) d \mu_{B}(x, y) \\
=\int_{\mathbb{R}^{k}} \int_{\mathbb{R}^{k}} \hat{\chi_{F}}(x) \hat{\chi_{G}}(y) \exp \left(-\frac{1}{2}\left(x^{T} A x+y^{T} C y+2 x^{T} B y\right)\right) d x d y .
\end{gathered}
$$

Taking the second partial derivative by $b_{i, j}$ and $b_{m, n}$, we get

$$
\begin{gathered}
H_{i, j, m, n}=\frac{\partial^{2}}{\partial b_{i, j} \partial b_{m, n}} \mu_{B}(F \times G) \\
=\int_{\mathbb{R}^{k}} \int_{\mathbb{R}^{k}} \hat{\chi_{F}}(x) \hat{\chi_{G}}(y)\left(x_{i} x_{m} y_{j} y_{n}\right) \exp \left(-\frac{1}{2}\left(x^{T} A x+y^{T} C y+2 x^{T} B y\right)\right) d x d y \\
=\frac{1}{(2 \pi)^{k}|\mathcal{A}|^{1 / 2}} \int_{F} \int_{G} \frac{\partial^{4}}{\partial x_{i} \partial x_{m} \partial y_{j} \partial y_{n}} \exp \left(-\frac{1}{2}(x, y)^{T} \mathcal{A}^{-1}(x, y)\right) d y d x .
\end{gathered}
$$

The fact that $|\mathcal{A}| \neq 0$, and the validity of using Parseval's Equality in the latter equations, follow from the non-singularity of the measure $\mu_{B}$.

Since the sets $F$ and $G$ are symmetric, the partial derivative of the function $B \mapsto \mu_{B}(F \times G)$ by each $b_{i, j}$ is equal to zero at the point $B=0$. In order to show that there is a local minimum at $B=0$, we need to know that $H$ is positive definite when $B=0$. Furthermore, by a change of variables, we see that it is sufficient to consider the special case when $A=C=I$. Hence, we need to show the positive definiteness of

$$
H_{i, j, m, n}=\frac{1}{(2 \pi)^{2 k}} L_{i, m} K_{j, n}
$$

where

$$
L_{i, m}=\int\left(x_{i} x_{m}-\delta_{i, m}\right) \exp \left(-\frac{1}{2} x^{T} x\right) d x
$$


and

$$
K_{j, n}=\int_{G}\left(y_{j} y_{n}-\delta_{j, n}\right) \exp \left(-\frac{1}{2} y^{T} y\right) d y
$$

Since $H=L \otimes K$, it is sufficient to show that $L$ and $K$ are negative definite, and clearly it is enough just to prove it for $L$.

Thus we desire to show that

$$
\sum_{i, m} L_{i, m} \alpha_{i} \alpha_{m}=\int_{F}\left(\left(\sum_{i} \alpha_{i} x_{i}\right)^{2}-\|\alpha\|_{2}^{2}\right) \exp \left(-\frac{1}{2} x^{T} x\right) d x<0
$$

for all $\alpha \neq 0$. But by a change of variables, it is sufficient to show

$$
\int_{F}\left(x_{1}^{2}-1\right) \exp \left(-\frac{1}{2} x^{T} x\right) d x<0
$$

for every convex symmetric set $F$ with compact closure.

To show this, we see this as

$$
\int_{-\infty}^{\infty}\left(x_{1}^{2}-1\right) \exp \left(-\frac{1}{2} x_{1}^{2}\right) \phi\left(x_{1}\right) d x_{1}
$$

where

$$
\phi\left(x_{1}\right)=\int_{\mathbb{R}^{k-1}} \chi_{F}\left(x_{1}, \ldots, x_{k}\right) \exp \left(-\frac{1}{2}\left(x_{2}^{2}+\cdots+x_{k}^{2}\right)\right) d x_{2} \ldots d x_{k}
$$

Since $\chi_{F}(x) \exp \left(-\frac{1}{2}\left(x_{2}^{2}+\cdots+x_{k}^{2}\right)\right)$ is log-concave in $\mathbb{R}^{k}$, the result of Prekopa and Leindler mentioned before the formulation of Theorem 2 implies that $\phi$ is also logconcave. Since $\phi$ is also symmetric, it follows that $\phi\left(x_{1}\right)=\phi_{1}(|x|)$, where $\phi_{1}$ is a decreasing function. Furthermore, since $F$ has compact closure, $\phi_{1}$ is non-constant. Hence in order to show that

$$
\int_{-\infty}^{\infty}\left(x_{1}^{2}-1\right) \exp \left(-\frac{1}{2} x_{1}^{2}\right) \phi\left(x_{1}\right) d x_{1}<0
$$

it is sufficient to show that for all $0<a<\infty$

$$
\theta(a)=\int_{-a}^{a}\left(x_{1}^{2}-1\right) \exp \left(-\frac{1}{2} x_{1}^{2}\right) d x_{1}<0
$$

The function under the latter integral has antiderivative $-x_{1} \exp \left(-\frac{1}{2} x_{1}^{2}\right)$, so the result follows.

Finally, we present one more argument showing that inequality (6) would be proved if one showed that the function from Theorem 2 had global minimum at 
Let $A=C=I$. Since the sets $F$ and $G$ are convex, their topological boundaries have zero Lebesgue measure. Let $\nu$ be standard Gaussian measure on $\mathbb{R}^{k}$. Then $\mu_{0}(F \times G)=\nu(F) \nu(G)$, whereas $\lim _{\lambda \rightarrow 1} \mu_{\lambda I}(F \times G)=\nu(F \cap G)$. To see this last assertion, note that

$$
\mu_{\lambda I}(F \times G)=\frac{1}{\left(\left(2 \pi\left(1-\lambda^{2}\right)\right)^{k}\right.} \int_{F} \int_{G} \exp \left(-\frac{1}{2\left(1-\lambda^{2}\right)}\left(x^{T} x-2 \lambda x^{T} y+y^{T} y\right)\right) d y d x
$$

which, making the substitution $x=u+v, y=u-v$

$$
=\frac{1}{\left(\pi\left(1-\lambda^{2}\right)\right)^{k}} \int_{\mathbb{R}^{k}} \int_{(F-u) \cap(u-G)} \exp \left(-\frac{u^{2}}{1+\lambda}-\frac{v^{2}}{1-\lambda}\right) d v d u .
$$

Now, if $u$ is not in the boundary of $F$ or the boundary of $G$, then it is easily seen that

$$
\lim _{\lambda \rightarrow 1} \frac{1}{(\sqrt{\pi}(1-\lambda))^{k}} \int_{(F-u) \cap(u-G)} \exp \left(-\frac{v^{2}}{1-\lambda}\right) d v=\chi_{F \cap G}(u) .
$$

Hence the last assertion follows by Lebesgue's law of dominated convergence.

It is clear now that, if the function $\mu_{B}$ has global minimum at zero then $\mu_{\lambda I}(F \times$ $G) \geq \mu_{0}(F \times G)$, and, hence, $\nu(F \cap G) \geq \nu(F) \nu(G)$. However, the question of whether the function from Theorem 2 has global minimum at zero remains open.

Acknowledgements. We would like to thank T. Schlumprecht, G. Schechtman and J. Zinn for bringing the problem to our attention and providing us with updated information including their unpublished results.

\section{REFERENCES}

1. J.A. Clarkson (1936), Univormly convex spaces, Trans. A.M.S. 40, 396-414.

2. K. Joag-dev, M. D. Perlman and L. Pitt (1983), Asociation of normal random variables and Slepian's inequality, Ann. Probab. 11, 451-455.

3. M.-L. T. Lee, S. Rachev and G. Samorodnitsky (1990), Association of stable random variables, Ann. Probab. 18, 1759-1764.

4. L. Leindler (1972), On a certain converse of Hölder's inequality II, Acta. Sci. Math. Szeged 33, 217-223.

5. W. Orlicz (1933), Uber unbedingte Konvergenz in Funktionen Raumen I and II, Studia Math. 4, 33-37 and 41-47.

6. L.Pitt (1977), A correlation inequality for gaussian measures of symmetric convex sets, Ann. Probab. 5, 470-474.

7. L. Pitt (1982), Positively correlated normal variables are associated, Ann. Probab. 10, 496499.

8. A. Prekopa (1973), On logarithmic concave measures and functions, Acta Sci. Math. (Szeged) 34, 335-343.

9. T. Schlumprecht, G. Schechtman and J. Zinn (1994), On the Gaussian measure of the intersection of symmetric convex sets, preprint.

10. C. Suquet (1994), Introduction a l'association, Pub. IRMA, Lille 34 (no. XIII), 3-19.

Division of Mathematics and Statistics, University of Texas at San Antonio, San Antonio, TX 78249, U.S.A.

E-mail address: koldobsk@ringer.cs.utsa.edu

Department of Mathematics, University of Missouri-Columbia, Columbia, MO 65211 